\title{
DESAFIOS TECNOLÓGICOS NA PRODUÇÃO DE PRODUTOS COM BAIXO TEOR DE LACTOSE
}

\section{Lactose-free dairy products: Challenges in the processing and quantification of lactose}

\author{
Virgínia Nardy Paiva ${ }^{l}$; Elisângela Ramieres Gomes ${ }^{l}$; Valéria Maria dos Santos ${ }^{l}$; \\ Rodrigo Stephani ${ }^{2}$; Antônio Fernandes de Carvalho ${ }^{1}$; Ítalo Tuler Perrone ${ }^{3 *}$
}

\section{RESUMO}

A lactose é o principal açúcar do leite, sendo hidrolisada em glicose e galactose através da ação da enzima lactase. Alguns indivíduos têm deficiência dessa enzima no organismo, portanto, são intolerantes à lactose. Com o intuito de atender as necessidades especiais desses consumidores o mercado de produtos zero lactose vem crescendo ao longo dos anos. Porém, ainda existe uma série de limitações industriais na fabricação desses alimentos. A produção de leite em pó deslactosado é um desafio para indústria, devido a ocorrência de problemas como adesão ao equipamento, gerando baixo rendimento industrial. Os métodos mais sensíveis e eficazes para quantificar a lactose residual são de alto custo, inviáveis à realidade industrial. Outras técnicas consomem muito tempo e não são capazes de distinguir individualmente os carboidratos. Os processos térmicos aplicados a esses alimentos causam danos sensoriais e nutricionais, visto que as unidades monossacarídeas da lactose são mais reativas à reação de escurecimento não enzimático. Além disso, os produtos deslactosados estão mais propensos a atividade proteolítica. Faz-se necessário a otimização dos processos de fabricação, visando a redução de tempo e custos operacionais, como também a obtenção de produtos de qualidade e seguros ao consumidor.

1 Universidade Federal de Viçosa, Departamento de Tecnologia de Alimentos (DTA), INOVALEITE, Viçosa, MG, Brasil.

2 Universidade Federal de Juiz de Fora, Departamento de Química (DQ), QUIMTEC, Juiz de Fora, MG, Brasil.

3 Universidade Federal de Juiz de Fora,Departamento de Ciências Farmacêuticas, Faculdade de Farmácia. Campus Universitário, Rua José Lourenço Kelmer, s/n, São Pedro, 36036-330, Juiz de Fora, MG, Brasil. E-mail: italotulerperrone@gmail.com

* Autor para correspondência.

Recebido / Received: 27/04/2018

Aprovado / Approved: 22/08/2018 
Palavras-chave: intolerância à lactose; produto deslactosado; lactose residual; reação de Maillard.

\begin{abstract}
Lactose is the main sugar in milk, being hydrolyzed in glucose and galactose through the action of the enzyme lactase. Some individuals are deficient of this enzyme, therefore, they are intolerant to lactose. In order to attend the special needs of these consumers, the free lactose product market has been growing over the years. However, there are still a number of industrial limitations on the manufacture of these products. The manufacture of lactose-free powdered milk is a challenge for industry, due to the occurrence of problems such as adhesion to the equipment, generating low industrial yield. The most sensitive and effective methods for quantifying lactose are high cost, making it unfeasible to the industrial reality. Other techniques are time consuming and cannot distinguish carbohydrates individually. The thermal processes applied to these foods cause sensorial and nutritional damages, because the monosaccharide units of lactose are more reactive to the reaction of non-enzymatic browning. Furthermore, these products are more prone to proteolytic activity. It is necessary to optimize the manufacturing processes, aiming at reducing time and operating costs, as well as obtaining quality and safe products to the consumer.
\end{abstract}

Keywords: lactose intolerance; lactose-free product; residual lactose, Maillard reaction.

\section{INTRODUÇÃO}

A lactose é um açúcar exclusivo e predominante do leite (WALSTRA et al., 2006). A digestão deste dissacarídeo no corpo humano ocorre através da ação da enzima lactase, que hidrolisa a lactose em glicose e galactose. Porém, algumas pessoas possuem deficiência ou ausência dessa enzima no organismo, causando o que conhecemos como "intolerância a lactose". O número de intolerantes vem crescendo, gerando uma expansão no mercado global de produtos deslactosados (PERATI et al., 2016). Atualmente é possível encontrar uma variedade de marcas e produtos lácteos deslactosados nos mercados, entretanto o processo de fabricação de alimentos como leite em pó zero lactose ainda é um desafio para indústria, devido a ocorrência de problemas de adesão ao equipamento, empedramento, escurecimento do pó, e baixo rendimento industrial (SHRESTHA et al., 2007; TORRES et al., 2017).

Há também uma série de limitações quanto aos atuais métodos analíticos para a quantificação da lactose residual em produtos deslactosados, pois muitas vezes as técnicas utilizadas não são industrialmente viáveis (PERATI et al., 2016). A maioria dos métodos conhecidos são demorados devido a extensa preparação de amostras, e incapazes de distinguir individualmente os carboidratos (GAMBELLI, 2017). Além disso, com a Resolução $n^{\circ}$ 135/08 de fevereiro de 2017, a partir do ano de 2019 para os alimentos serem considerados isentos a lactose, esses devem ter uma quantidade máxima de 0,1 gramas de lactose para cada 100 gramas ou mililitros do produto final, e os de baixo teor de lactose uma quantidade de até 1 grama por 100 gramas ou mililitros do alimento pronto para o consumo. Esse índice máximo de $0,1 \%$ de lactose 
é considerado seguro para os consumidores intolerantes, e foi baseado na experiência de países europeus (ANVISA, 2017). Nesse contexto, a redução do nível de lactose nos alimentos deslactosados aumenta ainda mais a importância na implementação de métodos analíticos eficientes e de baixo custo para a quantificação da lactose.

São necessários a utilização de equipamentos e técnicas simples, capazes de determinar os níveis de lactose com rapidez, em leite e/ou derivados lácteos (VASKOVA; BUCKOVA, 2016). Métodos gravimétricos, polarimétricos e enzimáticos, são exemplos de procedimentos antigos e pouco eficazes em mensurar baixas concentrações de lactose (GAMBELLI, 2017). O índice crioscópico é muito empregado para estimar o grau de hidrólise da lactose (CZARNOBAY et al., 2017). Para detectar baixos níveis de lactose com maior precisão utiliza-se a cromatografia líquida de alta eficiência (HPLC) ou cromatografia de troca aniônica de alto desempenho com detecção amperométrica pulsada (HPAE-PAD) (PERATI et al., 2016; SCHEPPINGEN-VAN et al., 2017; TRANI et al., 2017).

Durante o processamento e armazenamento, produtos deslactosados podem sofrer alterações químicas indesejadas. Estes são mais propensos a ocorrência de proteólise (TOSSAVAINEN; KALLIOINEN, 2008b), gerando sabor e odor desagradáveis, bem como alterações na viscosidade do produto (SANTOS et al., 2018). A aplicação de processos térmicos são necessários e objetivam principalmente em oferecer ao consumidor alimentos microbiologicamente estáveis, porém a intensidade e o tempo prolongado de exposição ao calor reduzem a qualidade das características nutricionais e sensoriais do produto (SILVA, 2000). A hidrólise da lactose em glicose e galactose além de conferir sabor adocicado aos derivados lácteos (ADHIKARI et al., 2010; SHENDURSE; KHEDKAR, 2016), aumenta consideravelmente a concentração de açúcares redutores, que com o efeito do tratamento térmico podem levar à formação dos produtos da reação de Maillard (TROISE et al., 2016). Por isso a ação do calor tem maior impacto aos produtos deslactosados, como problemas de escurecimento, sabor cozido e redução do valor nutricional (HOSHINO et al., 2009).

\section{REFERENCIAL TEÓRICO}

\section{Principais propriedades da lactose}

Conhecida como o açúcar do leite, a lactose é o principal carboidrato em sua composição, sendo que o leite contém apenas traços de outros açúcares (FOX; McSWEENEY, 1998; WALSTRA et al., 2006). É um nutriente primordial no desenvolvimento dos mamíferos, e seus teores variam entre as espécies; o leite de vaca contém entre $4,5 \%$ a $4,8 \%$ de lactose enquanto o leite humano possui cerca de 7\% de lactose (FOX; McSWEENEY, 1998; SHENDURSE; KHEDKAR, 2016).

A lactose consiste em um dissacarídeo composto por D-Glicose e D-Galactose unidos a partir de uma ligação $\beta$-1,4-glicosídica (WALSTRA et al., 2006). Possui em sua estrutura um grupo hemiacetal livre, portanto, é considerada um açúcar redutor, e consequentemente está susceptível a reação de escurecimento não enzimático, denominada reação de Maillard (FOX; McSWEENEY, 1998).

Sua configuração química não é estável, podendo mudar a forma de $\alpha$ para $\beta$, ou de $\beta$ para $\alpha$, esse fenômeno é denominado demutarrotação, ocorre quando a lactose está em solução (FOX; McSWEENEY, 1998). Apresenta baixa solubilidade (FENNEMA et al., 2010) e menor poder adoçante em relação a outros açúcares como a frutose, sacarose, glicose e galactose (RODRIGUES JUNIOR et al., 2016). Mediante ação enzimática a lactose pode ser hidrolisada em glicose 
e galactose (FOX; McSWEENEY, 1998). Esta reação de hidrólise é realizada pela enzima $\beta$-galactosidase (WALSTRA et al., 2006), conhecida popularmente como lactase (SANTIAGO et al., 2004).

\section{Produtos lácteos sem lactose}

Produtos lácteos com teor de lactose reduzido podem ser produzidos utilizando operações de nano e ultrafiltração, que removem aproximadamente $40 \%$ da lactose, ou pela hidrólise enzimática da lactose com a adição da enzima lactase (HARJU et al., 2012). Leite e derivados lácteos com lactose hidrolisada começaram a ser fabricados na década de 1970, quando se iniciou a comercialização das primeiras enzimas $\beta$-galactosidases (PANESAR et al., 2006). Aproximadamente $70 \%$ da população mundial têm algum grau de intolerância a lactose (MESSIA et al., 2007), por isso o mercado vem investindo em produtos deslactosados (SILVA, 2016), com o intuito principal de suprir as necessidades desses indivíduos que não conseguem catabolizar completamente a lactose em glicose e galactose (MORLOCK et al., 2014). Para o mercado brasileiro de produtos zero lactose estima-se um crescimento anual de $15 \%$ a $20 \%$ até o ano de 2020 , de acordo com pesquisa realizada pela Revista Indústria de Latícinios (2017).

Por se tratar de um nicho de mercado recente, as indústrias têm algumas limitações e desafios a serem enfrentados. A hidrólise da lactose leva a alterações na composição físco-química do produto que podem dificultar o processamento e causar modificações indesejáveis, desde as etapas de fabricação até o armazenamento (TORRES, 2016). Em comparação ao leite não hidrolisado, o leite com lactose hidrolisada é geralmente mais vulnerável a mudanças químicas (TOSSAVAINEN; KALLIOINEN, 2008a). Esses produtos deslactosados podem ter concentrações de lactose significativamente reduzidas, até abaixo de $0,1 \%$, porém para garantir ao consumidor intolerante um alimento seguro é preciso analisar o nível de lactose residual regularmente, através de métodos sensíveis e específicos para a detecção de lactose (GILLE et al., 2018).

\section{Alguns métodos analíticos utilizados para quantificar a lactose}

\section{HPLC-Cromatografia líquida de alta eficiência}

De modo geral, cromatografia é um método físico-químico de separação, baseada na migração lenta da amostra a ser analisada através de uma fase estacionária por intermédio de uma fase móvel (SKOOG et al., 2006). A cromatografia líquida de alta eficiência (HPLC) é uma forma rápida e eficaz de separação de componentes. O método ocorre através de pequenas colunas fechadas, onde a fase móvel líquida elui sobre a fase estacionária. A amostra interage tanto com a fase estacionária como com a fase móvel, resultando em uma maior variedade de recursos de separação e tornando a técnica mais versátil. Deve-se ressaltar que a amostra precisa ser solúvel a fase móvel, sem que haja a decomposição dos seus componentes à medida que seja transportada pela coluna (FIGUEIREDO, 2012).

Os resultados analíticos são obtidos rapidamente, por meio de alta pressão interna do sistema (FIGUEIREDO, 2012), devido ao uso de partículas menores no recheio da coluna cromatográfica (SKOOG et al., 2006). Uma bomba assegura ao sistema uma vazão contínua, fazendo com que a passagem do solvente e a separação dos constituintes da amostra sejam aceleradas. Podem ser empregados diversos tipos de detectores, comumente utiliza-se detectores ultravioleta, de fluorescência, índice de refração, eletroquímicos e de polarimetria (DEGANI et al., 1998). 
A HPLC é amplamente utilizada em diversas áreas; em lácteos é método de referência para detecção do teor de lactose em leite cru e em alguns produtos como creme de leite e leite em pó (MONTI et al., 2017). Possui grande vantagem de aplicação comparado a outros métodos devido a capacidade de detectar carboidratos de maneira direta (INDYK et al., 1996), alta sensibilidade e grande variedade de combinações entre as fases móvel e estacionária (SKOOG et al., 2006). Entre as desvantagens da HPLC estão a necessidade do uso de reagentes caros e de alta qualidade (GAMBELLI, 2017), como de equipamentos modernos e automatizados (FIGUEIREDO, 2012), que além de caros, exigem manutenção especializada (ADRIAN et al., 2000).

\section{HPAEC-cromatografia de troca aniônica de alto desempenho}

A cromatografia de troca iônica permite uma análise sensível e seletiva para baixas concentrações de lactose. Esse método é uma técnica de separação para compostos aniônicos, realizada em $\mathrm{pH}$ alto. Baseia-se no fato de que os carboidratos são ácidos fracos, e em pH elevado são parcialmente ionizados, podendo ser separados por mecanismos de troca aniônica (SCHEPPINGEN-VAN et al., 2017).

Em estudos, Montiet al. (2017) verificaram que esse método cromatográfico acoplado à detecção amperométrica pulsada, mostrou-se eficiente em mensurar baixas concentrações de lactose, permitindo quantificar concentrações de lactose, glicose e galactose inferiores a $1 \mathrm{mg} / 100 \mathrm{~g}$ em queijo maturado.

\section{Kits enzimáticos}

Os kits enzimáticos são baseados na hidrólise enzimática da lactose em seus respectivos monossacarídeos: glicose e galac- tose. Os kits disponíveis no mercado não são totalmente adaptados para produtos com baixos níveis de lactose. Para as amostras com alto teor de galactose e/ou glicose em relação à baixas concentrações de lactose, é necessário o uso de procedimentos corretivos que estipulem a verdadeira concentração de lactose, gerando um aumento de possíveis erros analíticos (TRANI et al., 2017).

\section{Índice crioscópico}

A análise de crioscopia é um método rápido, e por isso muito utilizado em laticínios para determinar o grau de hidrólise da lactose (PROZYN, 2010). É uma forma indireta de quantificar a lactose, essa técnica permite apenas verificar a intensidade da reação de hidrólise da lactose, através da depressão do ponto de congelamento do leite (TONETTI, 2015).

O índice crióscopio do leite está diretamente ligado a quantidade de sólidos solúveis presentes na solução, definido principalmente pela concentração em solução de lactose e cloretos (TRONCO, 1997). A hidrólise da lactose acarreta em um aumento da concentração de açúcares redutores no leite, aumentando a concentração dos componentes solúveis, por isso o índice criocópico é reduzido (TREVISAN, 2008). De acordo com Moreira et al. (2009), o grau de hidrólise é verificado a partir da equação 1 :

$G H=(350,877 \times$ Crioscopia final $)-$

(Crioscopia Inicial / 0,00285)

(Eq. 1)

\section{Efeitos tecnológicos}

\section{Reação de Maillard}

A reação de Maillard não sofre ação enzimática e ocorre na presença de açúcares redutores e grupamentos amino livres (TOSSAVAINEN, 2008), principalmente 
entre grupos amino de lisina em proteínas do leite (WALSTRA et al., 2006), produzindo produtos de glicação (LABUZA et al., 1998). Esta reação causa alterações sensoriais ao alimento, devido a formação de compostos de coloração escura, denominados melanoidinas, gera também redução do valor nutricional, através da reação de açúcares redutores com grupamentos amino livres provenientes de aminoácidos essenciais (FOX; McSWEENEY, 1998). Em relação a outros aminoácidos, a lisina é mais reativa por ter em sua composição grupamentos e-amino (SHIBAO; BASTOS, 2011). O tipo de açúcar redutor também afeta diretamente na velocidade da reação, sendo as hexoses consideradas mais reativas que os dissacarídeos (FRANCISQUINI et al., 2017).

Quando ocorre em organismos vivos, a reação de Maillard é intitulada Glicação (SHIBAO; BASTOS, 2011). A exposição a altas temperaturas, causada pelos tratamentos térmicos, e longos períodos de armazenamento de produtos lácteos aumentam os níveis de glicação (MILKOVSKA-STAMENOVA; HOFFMANN, 2017). Durante o armazenamento em temperatura ambiente a reação de Maillard pode progredir significativamente, refletindo na qualidade final do produto (TOSSAVAINEN; KALLIOINEN, 2008a). Para avaliar a intensidade do tratamento térmico e a estabilidade de armazenamento do produto, comumente observa-se a presença de marcadores específicos de processamento, sendo estes frutose, lactulose e furosina (MESSIA et al., 2007), a ocorrência do último, furosina, pode também estimar os danos causados à lisina devido a reação de Maillard (TOSSAVAINEN; KALLIOINEN, 2008a).

Derivados lácteos que têm baixo teor ou são isentos de lactose estão mais propensos a glicação em relação aos produtos lácteos convencionais, pois além da influência relacionada ao aquecimento provocado pelos processos térmicos, os delactosados se diferenciam por apresentarem em sua composição glicose e galactose, que são açúcares redutores mais reativos que a lactose. Tanto a glicose como a galactose são hexoses, portanto mais reativas à reação de Maillard quando comparadas à lactose, que é um dissacarídeo (MILKOVSKA-STAMENOVA; HOFFMANN, 2016).

Devido ao aumento da reatividade à reação de Maillard, os produtos originários de leite hidrolisado são mais escuros, e apresentam sabor caramelado acentuado (PERRONE et al., 2011). A coloração escura provocada pelos produtos da reação de Maillard é observada em lácteos como o leite UHT (HOSHINO et al., 2009) e o doce de leite (PERRONE, 2007; MOREIRA et al., 2009).

A fim de evitar as alterações sensoriais causadas por essa reação, aconselha-se hidrolisar a lactose após o tratamento térmico (MENDOZA et al., 2005). Em nota da Revista Indústria de Laticínios (2014), verifica-se que essa tecnologia já vem sendo utilizada como um diferencial por indústrias de lácteos no Brasil, justamente por se tratar de uma alternativa de processamento mais rápida que não altera os aspectos de cor e sabor do produto final. Esse processo é possível com a utilização de embalagens assépticas, onde se adiciona a lactase diretamente nas caixas de leite UHT, por exemplo. Resultando em uma redução da reação de Maillard e de custos operacionais, pois requer uma quantidade menor da enzima e reduz o tempo da hidrólise (HARJU et al., 2012).

\section{Atividade proteolítica}

Quando a enzima é adicionada após o processamento térmico do leite, é de extrema importância que a $\beta$-galactosidase seja pura e de alta qualidade, para evitar a ocorrência de atividade proteolítica indesejada, pois nessa situação não ocorre a inativação das enzimas pela ação do calor. A proteólise provoca alterações no sabor e odor do produto, 
com a formação de peptídeos e aminoácidos, podendo favorecer também a reação de Maillard (JANSSON, JENSEN et al., 2014).

Em comparação ao leite não hidrolisado, o leite com lactose hidrolisada possui uma maior atividade proteolítica (JANSSON, CLAUSEN, et al., 2014). Jansson, Jensen e seus colaboradores (2014) verificaram em estudos que as caseínas presentes no leite UHT com lactose hidrolisada foram consideravelmente afetadas pela atividade proteolítica, sendo as frações $\beta, \alpha_{\mathrm{s} 1}$ e $\alpha_{\mathrm{s} 2}$ da caseína as mais prejudicadas, já a $\kappa$-caseína e as proteínas do soro foram menos afetadas (TOSSAVAINEN; KALLIOINEN, 2008b). Em contrapartida, observou-se apenas uma moderada atividade proteolítica em leite UHT convencional não hidrolisado.

\section{Leite em pó deslactosado}

Denomina-se transição vítrea a transformação sólido-líquido de um material amorfo; essa mudança ocorre em uma determinada faixa de temperatura, conhecida como temperatura de transição vítrea (PERRONE et al., 2016). A hidrólise da lactose resulta em monossacarídeos de baixo peso molecular, glicose e galactose (SHRESTHA et al., 2007), ambos têm valores reduzidos de temperatura de transição vítrea (TTg) comparados a lactose, sendo 31 e $32{ }^{\circ} \mathrm{C}$ para glicose e galactose respectivamente, e $97^{\circ} \mathrm{C}$ para lactose (ROOS, 1993). A temperatura de transição vítrea desses açúcares influi diretamente na secagem de leite com lactose hidrolisada, dificultando o processo (SHRESTHA et al., 2007). Podendo resultar em fenômenos de aderência, colapso e cristalização durante a produção e armazenamento do alimento (FERNÁNDEZ et al., 2003). Quanto menor a temperatura de transição vítrea, maior a tendência de aglomeração e aderência (CHEN; PATEL, 2008).

O uso dos mesmos parâmetros operacionais de secagem da fabricação do leite em pó convencional se torna inviável para a produção de leite em pó deslactosado, pois nesse caso o pó é aquecido a uma temperatura acima da sua temperatura de transição vítrea, podendo ocorrer adesão e escurecimento (SHRESTHA et al., 2007). Além de causar desvantagens como o entupimento do equipamento e baixa produção, sendo importante o aprimoramento das condições de secagem para este tipo de produto (FIALHO et al., 2017).

\section{CONSIDERAÇÕES FINAIS}

$\mathrm{O}$ crescente interesse industrial em atender a demanda de consumidores intolerantes à lactose e a tendência em seguir dietas restritivas a este dissacarídeo, tende a aumentar a disponibilidade e variedade de produtos com baixo teor e/ou isentos de lactose nos mercados. Porém, existem algumas limitações industriais a serem enfrentadas, sendo necessário otimizar o processo de fabricação destes alimentos visando principalmente a redução de tempo, custos operacionais e a obtenção de produtos de alta qualidade, que atendam às exigências da legislação vigente.

Para isso ocorrer é fundamental a constante realização de pesquisas aplicadas à tecnologia, objetivando em métodos analíticos que possam ser inseridos à realidade industrial, e ao mesmo tempo sejam sensíveis e específicos ao mensurar a concentração de lactose residual. Bem como, a aplicação de novas rotas tecnológicas que melhorem as condições de processamento e armazenamento, e possam reduzir as alterações sensoriais e nutricionais indesejáveis ao produto final, levando em conta a importância do conhecimento científico sobre a influência da hidrólise da lactose na modificação das características físico-químicas do leite e seus derivados. 


\section{AGRADECIMENTOS}

Ao CNPq, a FAPEMIG e a CAPES pelo apoio financeiro aos projetos e as bolsas de produtividade da equipe do projeto.

\section{REFERÊNCIAS}

AGÊNCIA NACIONAL DE VIGILÂNCIA SANITÁRIA (ANVISA). Resolução RDC $n^{\circ} 135$, de 08 de fevereiro de 2017. Alteração da Portaria SVS/MS n ${ }^{\circ} 29$, de 13 de janeiro de 1998, que aprova o regulamento técnico referente a alimentos para fins especiais, para dispor sobre os alimentos para dietas com restrição de lactose. Diário Oficial da República Federativa do Brasil, Brasília, 09 fev. 2017. Disponível em: <http://pesquisa. in.gov.br/imprensa/jsp/visualiza/index. jsp? jornal $=1$ \&pagina $=44 \&$ data $=09 / 02 / 2017>$. Acesso em: 10 abr. 2018.

ADHIKARI, K. et al. Sensory characteristics of commercial lactose-free milks manufactured in the United States. LWT - Food Science and Technology, v. 43, n. 1, p. 113-118, 2010.

ADRIAN, J. et al. Analisis nutricional de los alimentos. Zaragoza: Acribia, 2000. 292p.

CZARNOBAY, M. et al. Cinética de hidrólise de lactose em leite de ovelha e leite de vaca. Revista CIATEC - UPF, v. 9, n. 1, p. 15-24, 2017.

CHEN, X. D.; PATEL, K. C. Manufacturing better quality food powders from spray drying and subsequent treatments. Drying Technology, v. 26, n. 11, p. 1313-1318, 2008.

DEGANI, A. L. G.; CASS, Q. B.; VIEIRA, P. C. Classificação pela forma física do sistema cromatográfico. Química Nova, v. 7, n. 7, p. 21-25, 1998.
FENNEMA, O. R.; SRINIVASAN D.; KIRK, L. P. Química de Alimentos de Fennema. Tradução Adriano Brandelli (et al.). 4 ${ }^{\mathrm{a}}$ ed. Porto Alegre: Artmed, 2010. 900p.

FERNÁNDEZ, E.; SCHEBOR, C.; CHIRIFE, J. Glass transition temperature of regular and lactose hydrolyzed milk powders. LWT Food Science and Technology, v. 36, n. 5, p. $547-551,2003$.

FIALHO, T. L. et. al.Lactose hydrolyzed milk powder: Thermodynamic characterization of the drying process. Drying Technology, v. 36, p. 922-931, 2017.

FIGUEIREDO, T. M. P. Validação de métodos analíticos. Determinação do teor de açúcar numa amostra de produto alimentar. 2012. 104f. Dissertação (Mestrado em Química) - Universidade de Coimbra, Coimbra, 2012.

FOX, P. F.; McSWEENEY, P. L. H. Dairy Chemistry and Biochemistry. 1998.

FRANCISQUINI, J. A. et al. Reação de Maillard: uma revisão. Revista do Instituto de Laticínios Cândido Tostes, v. 72, p. 4857, 2017.

GAMBELLI. L, Milk and Its Sugar-Lactose: A Picture of Evaluation Methodologies. Beverages, v. 3, p. 35, 2017.

GILLE, D. et al. Detection of lactose in products with low lactose content. International Dairy Journal, v. 83, p. 17-19, 2018.

H A R J U, M .; K A L LIOINEN, H . ; TOSSAVAINEN, O. Lactose hydrolysis and other conversions in dairy products: Technological aspects. International Dairy Journal, v. 22, Issue p. 104-109, 2012. 
HOSHINO, L. K. O. et al. Estudo da hidrólise na obtenção de leite lactose hidrolisado microfiltrado e avaliação de parâmetros físico-químicos para determinação da sua vida útil. In: CONGRESSO INTERINSTITUCIONAL DE INICIAÇÃO CIENTÍFICA, 3., 2009, Campinas. Anais eletrônicos... Campinas: IAC, 2009. Disponível em: <http:// www.iac.sp.gov.br/areadoinstituto/pibic/ anais/2009/Artigos/RE0901015.pdf>. Acesso em: 12 abr. 2018.

INDYK, H. E.; EDWARDS, M. J.; WOOLLARD, D. C. High performance liquid chromatographic analysis of lactosehydrolysed milk. Food Chemistry, v. 57, p. 575-580, 1996.

JANSSON, T.; CLAUSEN, M. R.; et al. Lactose-hydrolyzed milk is more prone to chemical changes during storage than conventional ultra-high-temperature (UHT) milk. Journal of Agricultural and Food Chemistry, v. 62, n. 31, p. 7886-7896, 2014.

JANSSON, T.; JENSEN, H. B.; et al. Chemical and proteolysis-derived changes during long-term storage of lactose-hydrolyzed ultrahigh-temperature (UHT) milk. Journal of Agricultural and Food Chemistry, v. 62, n. 46, p. 11270-11278, 2014.

LABUZA, T. P. et al. Maillard reactions in chemistry, food and health. Cambridge: Woodhead, 1998. 458p.

MENDOZA, A. et al. Chemical indicators of heat treatment in fortified and special milks. Journal of Agricultural and Food Chemistry, v. 53, p. 2995-2999, 2005.

MESSIA, M. C.; CANDIGLIOTA, T.; MARCONI, E. Assessment of quality and technological characterization of lactosehydrolyzed milk. Food Chemistry, v. 104, p. 910-917, 2007.
M I L K O V S K A - S TA M EN O VA, S .; HOFFMANN, R. Hexose-derived glycation sites in processed bovine milk. Journal of Proteomics, v. 134, p. 102-111, 2016.

M I L K O V S K A - S TA M EN O VA, S .; HOFFMANN, R. Influence of storage and heating on protein glycation levels of processed lactose-free and regular bovine milk products. Food Chemistry, v. 221, p. 489495, 2017.

MONTI, L. et al. Lactose, galactose and glucose determination in naturally "lactose free" hard cheese: HPAEC-PAD method validation. Food Chemistry, v. 220, p. 1824, 2017.

MOREIRA, K. M. M. et al. Produção de Doce de leite com teorreduzido de lactose por $\beta$-Galactosidase. Revista Acadêmica Ciências Agrárias Ambientais. v. 7, p. 375-382, 2009.

MORLOCK, G. E.; MORLOCK, L. P.; LEMO, C. Streamlined analysis of lactosefree dairyproducts. Journal of Chromatography, v. 1324, p. 215-223, 2014.

PANESAR, P. S.; PANESAR, R.; SINGH, R. S.; KENNEDY, J. F.; KUMAR, H. Microbial production, immobilization and applications of $\beta$-D-galactosidase. Journal of Chemical Technology and Biotechnology, v. 81, p. 530-543, 2006.

PERATI, P., DE BORBA, B., ROHRER, J. Determination of Lactose in Lactose-free Milk Products by High-performance Anionexchange Chromatography with Pulsed Amperometric Detection. Thermo Fisher Scientific, Application Note 248, 2016. Disponível em: $<$ https://www.thermofisher. com/content/dam/tfs/ATG/CMD/CMD $\% 20$ Documents / Application $\% 20 \& \% 20$ 
Technical $\% 20$ Notes/Chromatography/ Ion\%20Chromatography/AN-248-LactoseMilk-Products-HPAE-PAD-AN70236-EN. pdf>. Acesso em: 03 abr. 2018.

PERRONE, I. T. Tecnologia para a fabricação de doce de leite. Informe Agropecuário, v. 28, n. 238, p. 67-74, 2007.

PERRONE, I. T.; STEPHANI, R.; NEVES, B. S. Doce de leite: aspectos tecnológicos. Juiz de Fora, 2011. 186p.

PERRONE, I. T. et al. Soro em pó: estado vítreo e condições de operação do spray dryer. Revista do Instituto de Laticínios Cândido Tostes, v. 71, n. 2, p. 106, 2016.

PROZYN. Produtos com baixa lactose. Informação técnica, 2010.

REVISTA INDÚSTRIA DE LATICÍNIOS. Embaré lança leite zero lactose Camponesa. Revista Indústria de Laticínios, n. 109, julho/agosto 2014. Disponível em: <http:// www.revistalaticinios.com.br/download/ edicoes_anteriores/Revista_Industria_de Laticinios_109.pdf $>$. Acesso em: 10 jul. 2018.

\section{REVISTA INDÚSTRIA DE LATICÍNIOS.} Nova lactase traz inovação para produção de produtos zero lactose. Revista Indústria de Laticínios, n. 126, maio/junho 2017. Disponível em: $<$ http://www.revistalaticinios. com.br/download/edicoes_anteriores/IL-126bx-site.pdf $>$. Acesso em: 11 jul. 2018.

RODRIGUES JUNIOR, P. H. et al. FT-Raman and chemometric tools for rapid determination of quality parameters in milk powder: Classification of samples for the presence of lactose and fraud detection by addition of maltodextrin. Food Chemistry, v. 196, p. 584-588, 2016.
ROOS, Y. Melting and glass transitions weight carbohydrates of low molecular. Carbohydrate Research, v. 238, p. 39-48, 1993.

SANTIAGO, P. A. et al. Estudo da produção de $\beta$-galactosidase por fermentação de soro de queijo com kluyveromyces marxianus. Ciência e Tecnologia de Alimentos, v. 24, p. $567-572,2004$.

SANTOS, M. C. M. et al. Desenvolvimento de um modelo preditivo para identificação de perda de estabilidade e ocorrência de proteólise em leite UAT. Arquivo Brasileiro de Medicina Veterinária e Zootecnia, v. 70, n. 1, p. 247-253, 2018.

SCHEPPINGEN-VAN, W. B. et al. Selective and sensitive determination of lactose in lowlactose dairy products with HPAEC-PAD. Journal of Chromatography B, v. 1060, p. 395-399, 2017.

SHENDURSE, A.M.; KHEDKAR, C.D. Lactose, In: CABALLERO, B.; FINGLAS P.; TOLDRA, F. Encyclopedia of Food and Health. San Diego: Elsevier, 2016. p. 509-516.

SHRESTHA, A. K. et al. Water sorption and glass transition properties of spray dried lactose hydrolysed skim milk powder. LWT - Food Science and Technology, v. 40, n. 9, p. 1593-1600, 2007.

SHIBAO, J.; BASTOS, D. H. M. Produtos da reação de Maillard emalimentos: Implicações para a saúde. Revista de Nutrição, v. 24, n. 6, p. 895-904, 2011.

SILVA, J. A. Tópicos da tecnologia dos alimentos. São Paulo: Livraria Varela, 2000. $325 \mathrm{p}$.

SILVA, A. C. Desenvolvimento de doce de leite sem adição de sacarose e sem lactose. 
2016. 75 p. Dissertação (Mestrado Profissional em Ciência e Tecnologia do Leite e Derivados) - Universidade Federal de Juiz de Fora, Juiz de Fora, 2016.

SKOOG, D. A.; HOLLER, F. J.; NIEMAN, T. A. Principios de Análise Instrumental. $5^{\mathrm{a}}$ ed. Porto Alegre : Bookman, 2006. 836 p.

TONETTI, D. Leite semi-desnatado ultrapasteurizado com teor reduzido de lactose através do método enzimático. 2015. 35 p. Monografia (Especialização em Gestão da Qualidade na Tecnologia de Alimentos) - Universidade Tecnológica Federal do Paraná, Francisco Beltrão, 2015.

\section{TOSSAVAINEN, O. Heat-induced changes} in lactose hydrolysed milks. 2008. 76 p. Dissertation (Doctor of Science in Technology, Faculty of Chemistry and Materials Sciences) - Helsinki University of Technology, Espoo, Finland, 2008.

TOSSAVAINEN, O.; KALLIOINEN, H. Effect of lactose hydrolysis on furosine and available lysine in UHT skim milk. Milchwissenschaft, v. 63, n. 1, p. 22-26, 2008a.

TOSSAVAINEN, O.; KALLIOINEN, H. Furosine formation and proteolytic changes in carbohydrate reduced UHT milks. Milchwissenschaft, v. 63 , n. 3, p. 254-258, 2008 b.

TORRES, J. K. F. Caracterização e secagem em spray dryer de leite em pó integral com lactose hidrolisada. 2016. 49 p. Dissertação (Profissional em Ciência e Tecnologia do Leite e Derivados) - Universidade Federal de Juiz de Fora, Juiz de Fora, 2016.
TORRES, J. K. F. et al. Technological aspects of lactose-hydrolyzed milk powder. Food Research International, v. 101, n. February, p. 45-53, 2017.

TRANI, A. et al. Comparison of HPLC-RI, LC/MS-MS and enzymatic assays for the analysis of residual lactose in lactose-free milk. Food Chemistry, v. 233, p. 385-390, 2017.

TREVISAN, A. Influência de diferentes concentrações de enzima lactase e temperaturas sobre a hidrólise da lactose em leite pasteurizado. 2008. 60 p. Dissertação (Mestrado em Ciência e tecnologia de Alimentos) - Universidade Federal de Santa Maria, Santa Maria, 2008.

TROISE, A. D. et al. Evolution of protein bound Maillard reaction end-products and free Amadori compounds in low lactose milk in presence of fructosamine oxidase I. Food Chemistry, v. 212, p. 722-729, 2016.

TRONCO, V. M. Controle físico-químico do leite. Manual para inspeção da qualidade do leite. Santa Maria: UFSM, 1997. 208p.

VASKOVA, H; BUCKOVA, M. Measuringthe lactose content in milk. MATEC Web of Conferences, 2016. Disponível em: $<$ https:// www.matec-conferences.org/articles/ matecconf/abs/2016/39/matecconf cscc2016_05011/matecconf_cscc2016_ 05011.html $>$. Acesso em: 13 abr. 2018.

WALSTRA, P. et al. Dairy science and technology. New York: Taylor \& Francis Group, 2006. 768 p. 